\title{
Intrachain Ordering and Segregation of Polymers under Confinement
}

\author{
Youngkyun Jung, ${ }^{* \dagger}{ }^{\dagger}$ Juin Kim, ${ }^{\ddagger}$ Suckjoon Jun, ${ }^{\S}$ and Bae-Yeun Ha*,॥ \\ ${ }^{\dagger}$ Supercomputing Center, Korea Institute of Science and Technology Information, Daejeon 305-806, Korea \\ ${ }^{\ddagger}$ Department of Physics, Korea Advanced Institute of Science and Technology, Daejeon 305-701, Korea \\ ${ }^{\S}$ FAS Center for Systems Biology, Harvard University, Cambridge, Massachusetts 02138, United States \\ "Department of Physics and Astronomy, University of Waterloo, Waterloo, Ontario, Canada N2L 3G1
}

ABSTRACT: We study the relationship between intrachain ordering and segregation tendency of two polymers confined in a cylindrical space. We find the chains segregate spontaneously even outside de Gennes' linear-ordering scaling regime, in which each chain is a linear array of blobs. When the chains are weakly compressed against each other, linear ordering is well preserved and the chains remain segregated. On the other hand, for moderate compression, new

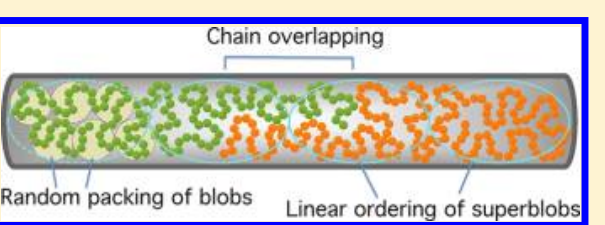
chain-ordering units emerge at intermediate length scales, within which blobs are randomly packed; yet these units (termed "superblobs") are linearly ordered, and the chains still segregate in the confined space. As the chains continue to be compressed, the linear regime disappears, but the chains can resist mixing effectively, more so in a more asymmetric space. We conclude that the linearly ordered E. coli chromosome is in the segregation regime.

\section{INTRODUCTION}

Considerable effort has been made recently to understand how chromosomes are spatially organized in cells. ${ }^{1-8}$ In particular, the entropic mechanism highlights the interplay between chain entropy and confinement: ${ }^{3-8}$ under the right conditions, two chromosomes partition or segregate spontaneously mainly driven by their chain entropy, especially in an elongated bacterial cell. Qualitatively speaking, under cylindrical confinement, chain molecules become more compactly space-filling and thus constrain each other better (see refs 10 and 11 for a polymer solution trapped in a cylindrical pore). This explains how entropic forces are brought about when individual chains experience anisotropic confinement. This entropic picture has offered quantitative insights into chromosome segregation, especially in rod-shaped E. coli cells, ${ }^{3-6}$ and challenged the conventional view that requires dedicated segregation machinery as in eukaryotes. ${ }^{12,13}$ It is worth emphasizing that the entropic picture is rather generic and largely independent of molecular details. ${ }^{6}$ It remains relevant under a variety of physical constraints (e.g., ring chain topology and chain crosslinking). ${ }^{3,6}$ A recent study even points out that various DNA binding proteins cooperate with the process of entropic segregation. $^{3}$

However, confined polymers have been much better understood at the single-chain level, whether they are flexible or stiff. ${ }^{14-20}$ For instance, (open) cylindrical confinement induces linear chain ordering ${ }^{6-21}$ and stiffen them. ${ }^{6,18,19}$ This polymer picture is consistent with the recent observation that the E. coli chromosome is linearly organized with high precision along the long axis, ${ }^{22,23}$ which would otherwise remain disordered (see refs 6 and 15 for theoretical explanations). Confinement not only reshapes individual chains ${ }^{21}$ but also has a nontrivial impact on how they interact (see ref 6 for some primitive discussion). Despite its significance in further exploiting the entropic mechanism, the relationship between intrachain organization and segregation remains to be explored. Is linear ordering required for chain segregation, as often assumed in the literature ? $^{3,5,10}$

Here, we study the relationship between intrachain ordering and segregation tendency of two self-avoiding chains confined in a closed cylindrical space (i.e., a cylinder capped at both ends), using molecular dynamics (MD) simulations as well as a phenomenological free energy approach; see the illustration in Figure 1. In particular, we consider the "internal distance" of each chain, $R_{i j}$, i.e., the direct distance between two monomers $i$ and $j$ on the same chain, as a function of $|i-j|$. We then relate it to the way the confined chains interact. Even though our study is limited to linear chains, its applicability goes beyond the linear case, since the effect of ring topology is shown to be mimicked in the linear case by appropriately reducing cylinder diameters. $^{6}$

When the chains are weakly compressed against each other, we find that their linear ordering is well preserved beyond $\xi \approx$ $D$, as in an open cylinder, and they remain segregated. ${ }^{3-6}$ Here, $\xi$ is the blob size or a length scale at which the crossover from the self-avoiding walk (SAW) to linear regime occurs and $D$ the diameter of the cylinder. ${ }^{21}$ For moderate compression, however, new ordering units emerge at some length scale $\zeta_{\|}$ $(>D)$ in the longitudinal direction, each consisting of several randomly packed blobs. Similarly to blobs in an open cylinder or in the weakly compressed case, these units are linearly ordered and are referred to as "superblobs". As a result, the

Received: November 25, 2011

Revised: March 6, 2012

Published: March 26, 2012 
chains segregate up to $80-90 \%$. As the chains continue to be compressed, linear ordering is eventually lost, but the chains can resist mixing effectively and remain segregated up to 70$80 \%$, more so in a more asymmetric space. While the chains do not mix, if linearly ordered, linear ordering is not a requirement for segregation.

Our results suggest that the linearly ordered E. coli chromosome $^{22,23}$ is in the spontaneous segregation regime. However, we do not attempt to resolve any discrepancy between the competing (active vs entropic) segregation mechanisms but content ourselves with general principles that are common to interacting chain molecules under confinement.

The article is organized as follows. The simulation procedure is outlined in Sec. II. Sec. III is mainly devoted to the analysis and interpretation of simulation results. In particular, the interrelationship between chain ordering and segregation is discussed in detail. Also, a free energy basis of chain segregation is presented. The entropic segregation of $E$. coli chromosomes is reconsidered in view of the interrelationship and the relevance of our polymer model is discussed.

\section{MOLECULAR DYNAMICS SIMULATIONS}

In our MD simulations, we use the bead-spring model of a polymer, which is characterized by two distinct interactions. (See subsection III.C for its relevance for the study of chromosome organization.) First, beads (among themselves and with the confining walls) interact via the fully repulsive Weeks-Chandler-Andersen (WCA) potential: ${ }^{24}$

$$
U_{\mathrm{WCA}}(r)= \begin{cases}4 \varepsilon\left[\left(\frac{\sigma}{r}\right)^{12}-\left(\frac{\sigma}{r}\right)^{6}+\frac{1}{4}\right] & \text { for } r<2^{1 / 6} \sigma \\ 0 & \text { otherwise }\end{cases}
$$

Here $\varepsilon$ and $\sigma$ represent the strength and range of the WCA potential, respectively; $r$ denotes the center-to-center distance between two beads, or the distance of the bead center from the confining cylinder minus $\sigma$. As a result, the monomer size $a \approx$ $\sigma$.

In addition, two neighboring beads are joined together through the finitely extensible nonlinear elastic (FENE) potential of the form

$$
U_{\mathrm{FENE}}(r)=-\frac{1}{2} k r_{0}{ }^{2} \ln \left[1-\left(\frac{r}{r_{0}}\right)^{2}\right]
$$

where the molecular spring constant $k=30 \varepsilon / \sigma^{2}$ and the maximum bond length $r_{0}=1.5 \sigma .^{25}$ This interaction ensures chain connectivity.

We integrated Newton's equations of motion using the velocity Verlet algorithm with an integration time step $\delta t=$ $0.01 \tau$, where $\tau=\sigma(m / \varepsilon)^{1 / 2}$ is the characteristic time scale with bead mass $m=1$. A Langevin thermostat with the damping constant $0.1 \tau^{-1}$ was used to keep the system at the fixed temperature $T=1.0 \varepsilon / k_{\mathrm{B}}$, where $k_{\mathrm{B}}$ is the Boltzmann constant (see ref 19 for details). To obtain reliable chain statistics, we carried out 200 independent simulations corresponding to different initial chain conformations. We first performed $10^{8}$ integration steps in order for the chain to equilibrate for a given initial conformation; after equilibration, we ran additional $10^{8}$ integration steps and obtained a data point every $10^{4}$ steps.

\section{RESULTS}

A. Chain Ordering and Segregation. First, we have examined chain structures under a varying degree of confinement and longitudinal compression. In this work, the chain structure is quantified in terms of the internal distance $R_{i j}=$ $\left[\left\langle\mathbf{R}^{2}(|i-j|)\right\rangle\right]^{1 / 2}$, the direct distance between two monomers $i$ and $j$ on the same chain, as a function of $|i-j|$, i.e., the contour length in units of $a$, where $\langle\ldots\rangle$ is an ensemble average or an average over simulations (see section II for details). Figure 1 shows our simulation results for $R_{i j}$ for $D=14$ (a) and $D=20$ (b). Here and below, all lengths are measured in units of $a$, unless otherwise stated. Different colors are used to represent a varying degree of longitudinal compression (see the legend) or a range of the volume fraction of monomers denoted as $\phi$. This consideration allows us to probe internal chain statistics under various conditions. Here, we relate single-chain properties such as $R_{i j}$ to interchain organization. The main advantage is that our results will not be complicated by partial overlapping, which would make each chain inhomogeneous, as illustrated in the inset in Figure 1. To this end, we first trap each chain in a closed cylinder of length $L$ and calculate $R_{i j}$. In our analysis of interchain organization, two chains are confined in a cylinder of length $2 L$ and the overlap distance $\lambda$ is measured, where $\lambda$ is the distance between the two farthermost monomers on different chains as described in the inset of Figure 1 (also see Figure 2a for an alternative interpretation).

A few distinct regimes are identified and labeled: the selfavoiding walk (SAW) at length scales $<\xi$, random walk (RW) at intermediate length scales, and linear regimes outside the RW regime, which are depicted by dashed lines with different slopes $3 / 5,1 / 2$, and 1 , respectively. ${ }^{26}$ In addition to the representative dashed line, we have included a thin dashed line to highlight the RW regime for some parameter choices in Figure $1 \mathrm{a}, \mathrm{b}$ - its emergence is clear. For sufficiently small $L$ [e.g., the blue curve with symbols in (a)], however, the RW regime is not clearly shown. This can be attributed to closed-confinement effects, which tend to make $R_{i j}$ saturated beyond a special value of $\mid i-$ jl.

For moderate compression, as illustrated in the top panel, all the three regimes are realized (e.g., the light blue curve in (a) and the dark yellow curve in (b), both with symbols). For weak compression (the first five curves in (a) and the first two curves in (b), from the top), the RW regime is missing, while for strong compression, the linear regime disappears (the curves with filled symbols). The length scale $\xi$ at which the crossover from the SAW to RW or linear regime occurs naturally defines structural units known as "blobs" and is smaller for larger $\phi$ (the semidilute scaling law $\xi \approx \phi^{-3 / 4}$ will apply to the confined chain case as long as $\xi<D) ;{ }^{21}$ a few blobs are highlighted in the top panel. Chain segments within a blob are not perturbed by confinement or other segments outside the blob. ${ }^{21}$ In other words, self-avoidance is unscreened.

The physical picture emerging from our results in Figure 1 is as follows: for weak compression, linear ordering is well preserved beyond $\xi \approx D$, in the sense that the RW regime is not realized, as in an open cylinder. ${ }^{21}$ As a result, $\xi \approx D$ is the only crossover length scale that characterizes the chain conformation. The appearance of the RW regime for moderate compression is a natural consequence of breakage of each blob into smaller ones, as $\phi$ increases $^{20}$ (also see ref 27 for a 


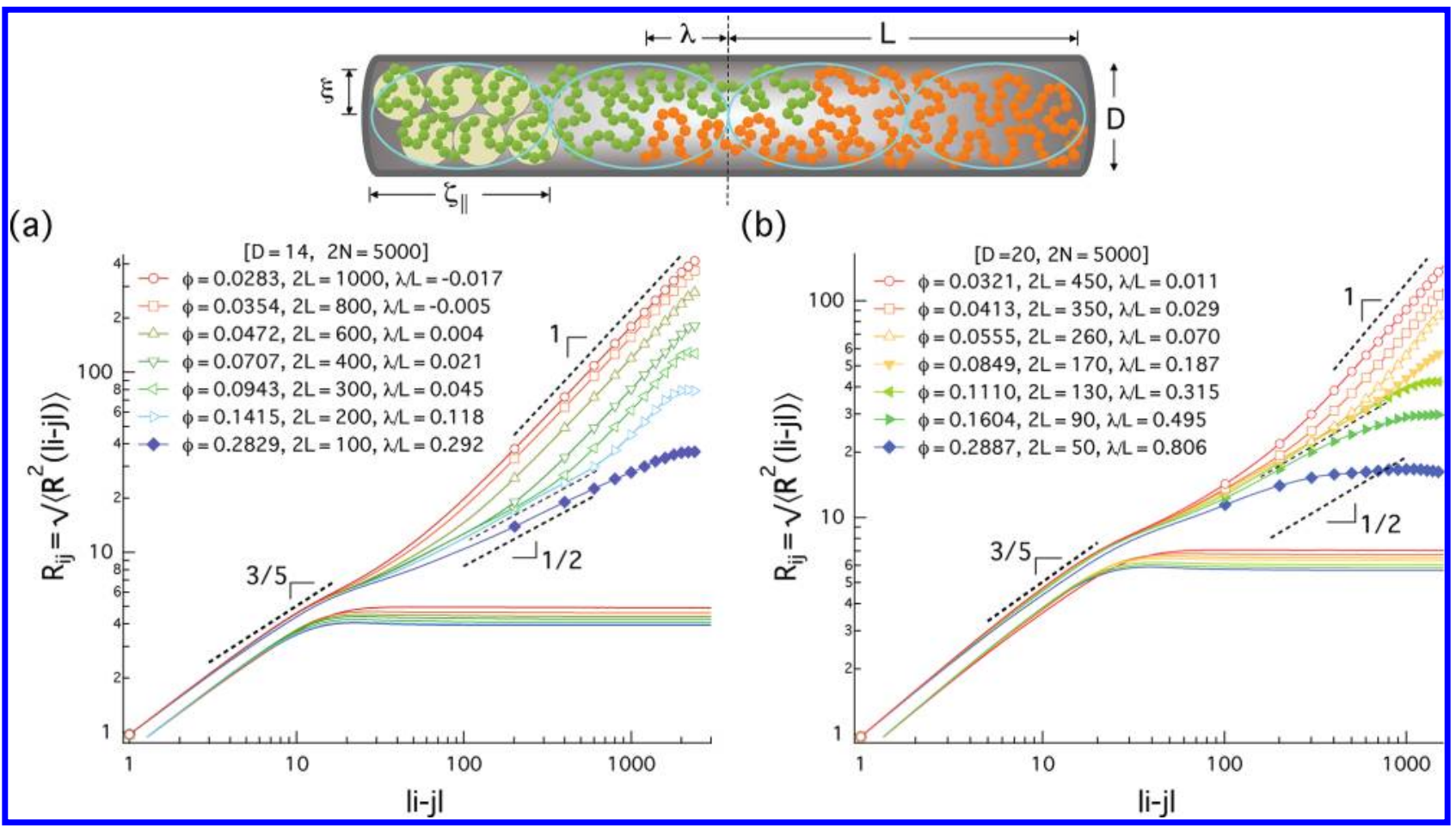

Figure 1. Internal distance of a confined chain $R_{i j}=\left[\left\langle\mathbf{R}^{2}(|i-j|)\right\rangle\right]^{1 / 2}$ (solid lines with symbols) and its relationship with $\lambda / L$, the fractional overlap distance between two chains (specified in the legend) for $D=14$ in (a) and $D=20$ in (b) in units of $a$. The dashed lines, with the slopes 3/5, 1/2, and 1 , depict the self-avoiding walk (SAW) within $\xi$, random walk (RW) at intermediate lengths, ${ }^{26}$ and linear regimes, respectively. If the confined chain is weakly compressed longitudinally, it is linearly ordered beyond the blob size $\xi \approx D$, as in an open cylinder, and the RW regime is missing. For intermediate compression, as illustrated in the top panel, "superblobs" emerge as ordering units at some length scale denoted as $\zeta_{\|}$, within which blobs are randomly packed (a few blobs are highlighted). For strong compression, the linear regime is suppressed (i.e., $\zeta_{\|} \approx L$ ). For the entire range shown, the RW regime is not clearly seen in the transverse component $R_{i j}^{\perp}=\left[\left\langle\mathbf{R}_{\perp}^{2}(|i-j|)\right\rangle\right]^{1 / 2}$ (the bottom lines without symbols). In all cases that display the linear regime (solid lines unfilled symbols), the chains are (almost) completely segregated. Even when linear ordering is lost, they segregate up to $70-80 \%$, more so in a more asymmetrical space. Under anisotropic confinement, chain segregation in the RW regime is sensitive to the aspect ratio $k=D / 2 L$.

similar issue under spherical confinement). Compression diminishes chain ordering in the sense that it makes "slower" the crossover to the linear regime at some length scale denoted as $\zeta_{\|}(>D)$. The crossover length $\zeta_{\|}$defines new chain-ordering units, referred to as "superblobs", each consisting of randomly packed blobs, as illustrated in Figure 1. Note the asymmetrical shape of the superblob, which is elongated in the longitudinal direction. In this case, linear ordering is preserved for length scales $>\zeta_{\|}>D$. As the chains continue to be compressed, $\zeta_{\|}$ increases and becomes comparable to the chain size eventually. The linear regime then disappears.

The unique anisotropy of cylindrical confinement is responsible for the crossover from the RW to linear regime at the length scale $\zeta_{\|}$, which is missing under spherical confinement. $^{27}$ This necessitates the introduction of new ordering units (called superblobs in this work). To capture the anisotropy better, it proves useful to consider the internal distance in the transverse direction $R_{i j}^{\perp}=\left[\left\langle\mathbf{R}_{\perp}^{2}(|i-j|)\right\rangle\right]^{1 / 2}$, where $\mathbf{R}_{\perp}(|i-j|)$ is the transverse component of $\mathbf{R}(|i-j|)$. For the entire parameter range shown in Figure 1, the RW regime is not seen clearly in the transverse component (see the bottom curves without symbols). Even in the RW regime, the confine chain is transversely restricted.

Also included in the legend is the fractional overlap distance $\lambda / L$, which describes interchain organization or "effective" chain miscibility ${ }^{28}$ (recall that $\lambda$ is the distance between the two farthermost monomers on different chains). In all cases that display the linear regime (curves with open symbols), the chains are segregated completely (for weak compression) or up to $80-90 \%$ (for moderate compression). However, the emergence of the linear regime is not a requirement for entropic segregation, as postulated in the literature. ${ }^{3,5,10}$ In fact, the chains resist mixing effectively, even when the linear regime is completely lost, as indicated by the bottom curve with symbols in (a) or the fifth one in (b); chain miscibility is still low around $30 \%$. This observation is analogous to the recent finding that two polymers in a melt can segregate in a cylindrical box. ${ }^{29}$ (In the melt, $\xi \approx a$ and the SAW regime is suppressed.) Similarly, it was shown earlier that ring polymers in a melt segregate. ${ }^{30}$ This is understandable in light of the recent finding that under cylindrical confinement ring polymers segregate better than the corresponding linear chains. ${ }^{6}$

As the chains continue to be compressed against each other, their linear regime will diminish in size and they mix or overlap better. The free energy basis for the crossover from the mixed to segregated state has been illusive. (See refs 10 and 20 for the difficulty with a free energy approach and subsection B for our attempt.) Crudely speaking, the free energy cost for chain backfolding inside a superblob becomes comparable to that for chain overlapping (also see ref 6 for relevant discussion). This will enhance (effective) chain miscibility.

Finally, compare (a) and (b) for the sensitivity of interchain organization to $D$, especially for $\phi \approx 0.29$ (the bottom curves with symbols in both cases). In both cases, each chain is a RW 


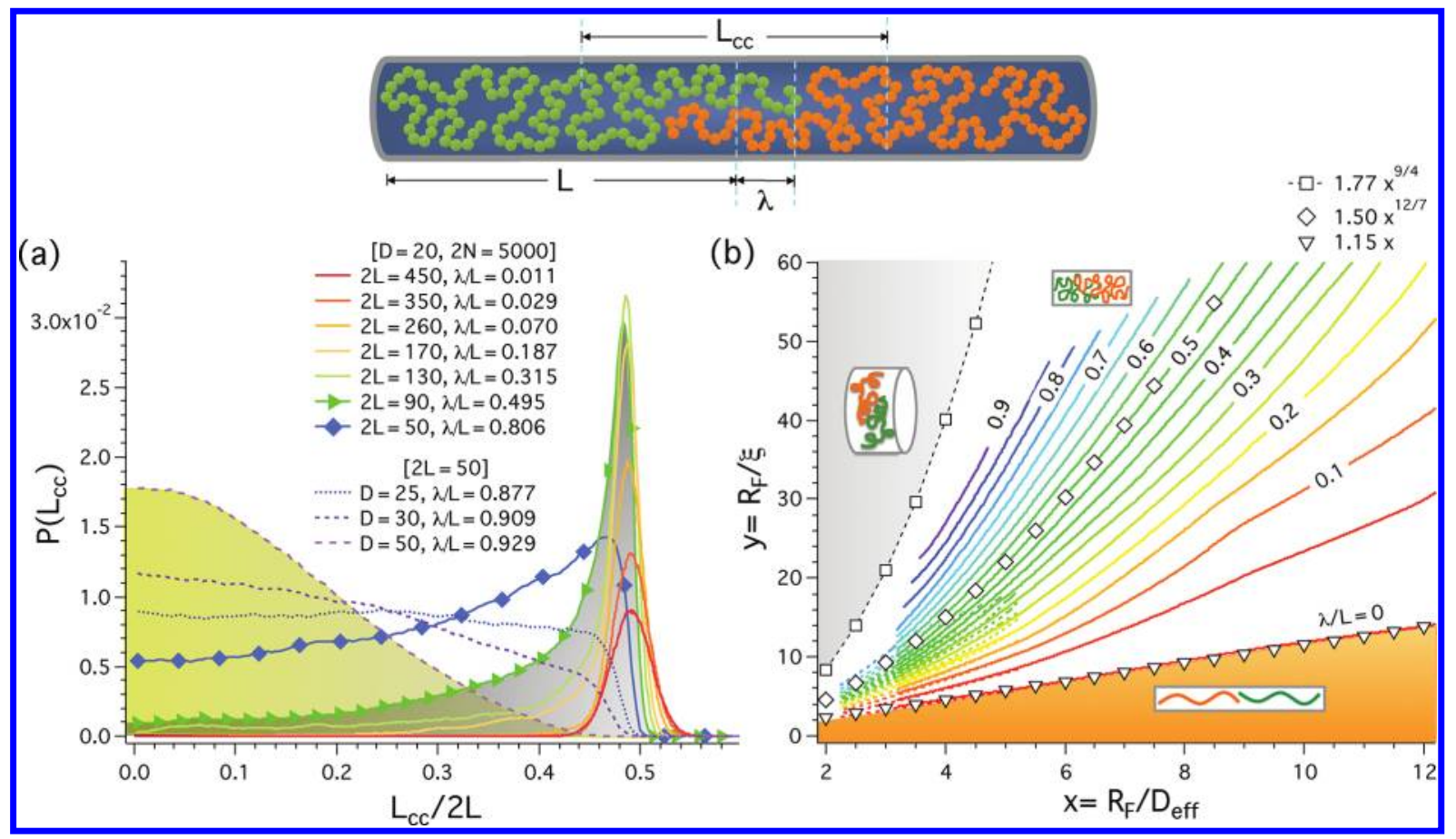

Figure 2. (a) Distribution of the center-to-center distance $L_{\mathrm{cc}}$ of two confined chains $P\left(L_{\mathrm{cc}}\right)$ (a) and chain miscibility (b). (The miscibility diagram is reproduced from ref 6 with modifications by permission of the Royal Society of Chemistry.) The color scheme in these plots is comparable to that used in Figure $1 \mathrm{~b}$. For the well-segregated cases (solid lines without symbols), $P\left(L_{\mathrm{cc}}\right)$ has a narrow peak at $L_{\mathrm{cc}} / 2 L \approx 0.5$. On the other hand, $P\left(L_{\mathrm{cc}}\right)$ for the much mixed cases (lines with filled diamonds) is broader. In the most miscible case $k=D / 2 L=1$ (the dashed line in purple); $P\left(L_{\text {cc }}\right)$ is a Gaussian distribution centered at $L_{\mathrm{cc}}=0$. In the diagram in (b), $D_{\text {eff }}=D$ for linear chains (solid lines), while for ring polymers, $D$ eff $=D / \sqrt{ } 2($ dashed lines). ${ }^{6}$ The dotted line with open squares describes the symmetrical case of $k=D / 2 L=1$ for which $y=1.77 x^{9 / 4}$. Beyond this, the confined space resembles a closed "slit". We have also included the theoretical prediction of the boundary curve $y=1.50 x^{12 / 7}$ between the segregated and mixed regimes 3,10 (see open "diamonds"). The prefactor has been chosen so that the boundary curve collapses on one of the contours. It best fits the line describing $\lambda / L=0.5$. In addition, we note that the $\lambda / L=0$ line is best fit by $y=1.15 x$ (open inverted triangles).

beyond $\xi \approx \phi^{-3 / 4}$, which is roughly the same for both curves, up to the entire contour length in the longitudinal direction. However, $\lambda / L$ is significantly different between the two cases. If the chains are much segregated in (a), they are almost completely mixed in (b). Thus, the smaller $D$ case in (a), more constrained in the transverse direction, shows a stronger segregation tendency. Even for RW chains, segregation can be sensitive to the aspect ratio of the confined space.

Our results in Figure 1 are statistical averages over many realizations of chain conformations. To understand chain miscibility beyond the average behavior, we have plotted in Figure $2 \mathrm{a}$ the distribution of the center-to-center distance $L_{\mathrm{cc}}$ for $D=20$, denoted as $P\left(L_{\mathrm{cc}}\right)$, as a function of $L_{\mathrm{cc}} / 2 L$. This is normalized so that $\int_{0}^{2 L} P\left(L_{\mathrm{cc}}\right) \mathrm{d} L_{\mathrm{cc}}=1$. Here we have employed the same color scheme used in Figure $1 \mathrm{~b}$ to represent various cases. Also note that the curves describing $\lambda / L \approx 0.5$ (green with symbols) and $2 L=D$ (purple dashed) are highlighted with a shade, since they represent special boundaries in the diagram in (b). As shown in the figure, for the much segregated case (curves without symbols in this plot), $P\left(L_{\mathrm{cc}}\right)$ has a peak at $L_{\mathrm{cc}} /$ $2 L \approx 0.5$ or $L_{\mathrm{cc}} \approx L$, meaning that the two chains are well segregated, consistent with our corresponding results for $\lambda / L$; also, the width of the peak is narrow and is about $10 \%$ of the cylinder length. Similarly, we have shown that $P\left(L_{\mathrm{cc}}\right)$ for $D=14$ has a peak at $L_{\mathrm{cc}} \approx L$ for the parameters used in Figure 1a (the data not shown here).

In contrast, $P\left(L_{\mathrm{cc}}\right)$ for the much mixed case (the solid lines with filled symbols) is broader. Nevertheless, the most probable state corresponding to the peak of $P\left(L_{\mathrm{cc}}\right)$ is still in the segregated regime, i.e., $L_{\mathrm{cc}} / 2 L \approx 0.5$, even when $\lambda / L>0.5$. However, there is no contradiction between the two pictures. In the overlapping region, each chain is better aligned than in the homogeneous region. This means that the farthermost monomer in green can reach deeper into the right-half space than indicated by $P\left(L_{\mathrm{cc}}\right)$. Furthermore, $\lambda / L=0.5$ has special meaning in chain miscibility. It separates between the segregated and mixed regimes, as shown below. This seems to be consistent with the miscibility picture based on $\lambda / L$ (and even with the picture $P\left(L_{\mathrm{cc}}\right)$ presents, as long as it is interpreted correctly).

The three dotted or dashed lines in Figure 2a show how $P\left(L_{\mathrm{cc}}\right)$ evolves as the confined space becomes symmetrical or as the aspect ratio $k=2 L / D$ approaches one. In our simulations, we increased $D$ from $D=20$ up to 50 , while keeping $2 L=50$. For $D=25, P\left(L_{\mathrm{cc}}\right)$ is almost flat, meaning that the free energy cost is comparable for random packing and for chain overlapping. As $k \rightarrow 1$, chain directionality is lost and $P\left(L_{\text {cc }}\right)$ becomes a Gaussian function centered at the origin (the purple curve with a shade). The confined chains are most miscible for $k=1$, as supported by our miscibility diagram in Figure $2 \mathrm{~b}$ (also see below). However, this has to be understood with some caution, since the distribution is broad. In this case, the most probable state corresponding to $L_{\mathrm{cc}}=0$ (i.e., completely mixed) does not necessarily coincide with what we expect from chain miscibility on average. Nevertheless, the two chains under isotropic confinement are well mixed, i.e., more than $90 \%$.

Displayed in Figure $2 \mathrm{~b}$ is an effective miscibility diagram in the plane of $x=R_{\mathrm{F}} / D_{\text {eff }}$ and $y=R_{\mathrm{F}} / \xi$, where the fractional 
overlap distance $\lambda / L$ is specified. Here, $R_{\mathrm{F}}=$ constant $\times N^{3 / 5}$ (constant $=1.1$ for linear and 0.87 for ring chains); $D_{\text {eff }}=D$ for linear chains (solid lines), while for ring polymers, $D_{\text {eff }}=D / \sqrt{ } 2$ (dashed lines). ${ }^{6}$ The agreement between the linear and ring chain means that ring-topology-enhanced segregation can be mimicked in the linear case by reducing $D$ as $D \rightarrow D / \sqrt{ } 2$ (see ref 6 for details). The color schemes used in Figure 2a,b and in Figure $1 \mathrm{~b}$ are comparable to each other; for instance, the red line represents $\lambda / L=0$ and the blue line $\lambda / L=0.8$. In earlier scaling approaches, ${ }^{3,10}$ the mixed and segregated regimes are separated by the curve $y=x^{12 / 7}$. To test this, we have plotted the curve $y=1.50 x^{12 / 7}$ (see unfilled diamonds). The prefactor has been chosen so that the boundary curve collapses onto one of the contours. Our analysis indicates that the curve best fits the line describing $\lambda / L=0.5$. See the green curve with a shade in Figure $2 \mathrm{a}$ for the corresponding $P\left(L_{\mathrm{cc}}\right)$. In view of this, this fitting curve describes the crossover boundary between the segregated and mixed regimes. According to our results in Figure $1 b$, linear ordering is completely lost on this curve.

In addition, we note that the $\lambda / L=0$ line is best fit by $y=$ $1.15 x$ (inverted triangles). The prefactor in this relation $(>1)$ is consistent with the observation that the confined chains resist mixing, even when they are weakly compressed against each other, and thus $\xi$ is somewhat smaller than $D$. Finally, we have included the curve $y=1.77 x^{9 / 4}$ (the dotted line with squares) that represents the isotropic case of $D=2 L$, for which the chains are most miscible [recall $P\left(L_{\mathrm{cc}}\right)$ is Gaussian centered at $\left.L_{\mathrm{cc}}=0\right]$. For $k \approx 1$, however, the notion of the overlap distance becomes obscure, since the distinction between longitudinal and transverse directions becomes arbitrary. Nevertheless, our results in Figure 2a,b clearly indicate that the confined chains are well mixed. Beyond this curve, however, the confined space resembles a closed slit, as illustrated by a cartoon on the left in Figure $2 b$.

B. Scaling Approach to Chain Segregation. Our results for $R_{i j}$ in Figure 1 indicate that compressed chains resist mixing as long as they are overall linearly ordered, i.e., $\zeta_{\|} \ll L$. However, this finding is not as obvious to interpret as it may seem, especially on a free energy basis. The main difficulty is that a free energy approach (at the mean-field level) is not always reliable and often leads to incorrect results. ${ }^{10,21}$ Even for the case of a single confined chain, there has been some confusion and the correct free energy approach, known as a renormalized Flory approach, has only recently been obtained (see ref 20 and references therein). Here we extend this approach as a free energy basis for segregation in the linear regime. To this end, we compare the two limiting cases: "segregated" and "mixed," as illustrated in Figures 3a and 3b, respectively.

First, recall that the free energy of a single chain in an open cylinder is given by

$$
\frac{\mathcal{F}(L)}{k_{\mathrm{B}} T}=A \frac{L^{2}}{(N / g) D^{2}}+B \frac{D(N / g)^{2}}{L}
$$

where $A$ and $B$ are (nonuniversal) constants of order 1 and $g \approx$ $D^{5 / 3}$ is the number of monomer per blob. ${ }^{21}$ Recall all lengths are estimated in units of $a$. Here $L$ should be interpreted as the longitudinal size of the confined chain, which coincides with the cylinder length. The free energy in eq 3 leads to correct chain sizes, and confinement free energy, and effective spring constants. This approach is based on the assumption that the confined chain is in the linear regime and remains valid unless

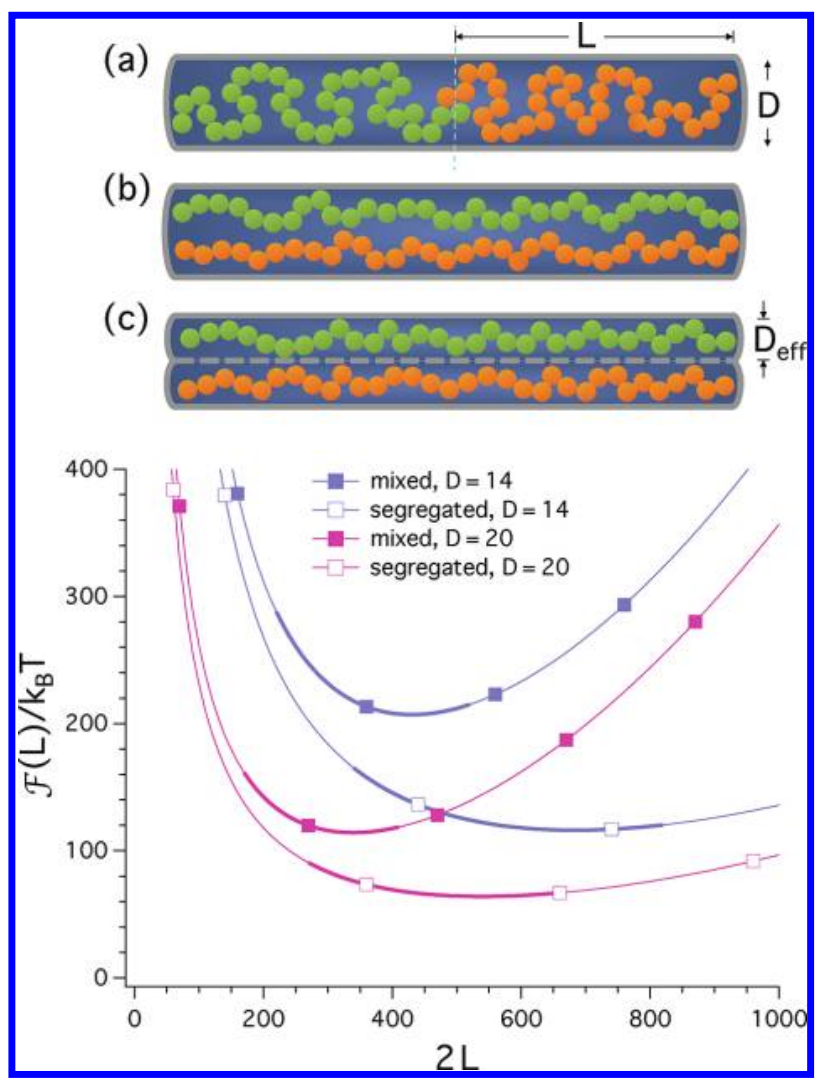

Figure 3. Free energy basis for chain miscibility in the linear regime (i.e., $\left.\zeta_{\|} \ll L\right)$; for visual clarity, the weakly compressed case $\left(\zeta_{\|} \approx \xi\right)$ is illustrated in the top panel. We compare the Flory free energy $\mathcal{F}(L)$ of a segregated state (a) with that of a mixed state (b), as a function of $L$, for $D=14$ and 20; in both cases, $N=2500$. To this end, we map (b) onto (c) for which $D_{\text {eff }}=D / \sqrt{ } 2$ is the effective diameter of an imaginary tube enclosing each chain. As shown in this figure (the bottom panel), mixing is disfavored by free energy. As $L$ decreases, the difference is diminished, and the chains are more miscible for smaller $L$. Our Flory approach is known to be accurate for $0.5 L_{0}<L<1.2 L_{0}{ }^{20}$ where $L_{0} \sim N D^{-2 / 3}$ is the unperturbed chain size in the equivalent open cylinder case. ${ }^{21}$

the chain is too much compressed longitudinally. Reference 20 suggests that it is valid for $0.5 L_{0}<L<1.2 L_{0}{ }^{20}$

To understand the applicability of $\mathcal{F}(L)$ in eq 3 for $0.5 L_{0}<L$ $<1.2 L_{0}$, note that at the lower bound, i.e., $L_{\text {lower }}=0.5 L_{0}, \xi \approx$ $0.6 D$, if we take $\xi=D$ for $L=L_{0}$, as assumed in de Gennes' scaling approach. ${ }^{21}$ The RW regime is not realized for this range of $L$, i.e., the range $0.5 L_{0}<L<1.2 L_{0}$ represents weak compression, consistent with the illustrations in the top panel in Figure 3. As $L$ decreases, blobs will break into smaller ones, but only for sufficiently small $L$, the RW regime will emerge, as described in Figure 1. In this case, our free energy approach is less accurate quantitatively. This is, however, a general feature of a Flory-type approach, which is formulated in terms of a single-order parameter, i.e., $L$ in our case. Here, we argue that this limitation will not invalidate our free energy comparison between the segregated (a) and mixed states (b), since it will influence both cases similarly.

By requiring that the free energy of segregated chains is extensive with the number of chains for given $\phi$, we can readily 
write down the free energy of the two chain system in (a), each occupying a half space of length $L$, as

$$
\mathcal{F}_{\text {seg }}(L) \approx 2 \mathcal{F}_{\text {linear }}(L)
$$

On the other hand, a recent study suggests that under cylindrical confinement a ring polymer can map onto a "parallel connection" of two linear chains, each occupying an imaginary cylinder of reduced diameter $D_{\text {eff }}=D / \sqrt{2}$, as illustrated in (c). On the basis of this, we argue that the free energy of two mixed chains in (b) is well approximated by that in (c). For visual clarity, the chains in de Gennes' linearly ordered scaling regime are illustrated in the top panel.

Following ref 6, we have

$$
\frac{\mathcal{F}_{\text {mix }}(L)}{k_{\mathrm{B}} T} \approx \hat{A} \frac{L^{2}}{(N / g) D^{2}}+\hat{B} \frac{D(N / g)^{2}}{L}
$$

where $\hat{A}=2^{13 / 6} A$ and $\hat{B}=2^{13 / 6} B$. Here, the parameters $g$, $N$, and $D$ in the second term on the right-hand side are for the corresponding linear case.

In Figure 3 (the bottom panel), we compare the segregated (a) and mixed states (b) as a function of $2 L$ the cylinder length using two choices of $D: D=14$ and 20. Also, we have chosen $A$ $=B=1$ as well as $2 N=5000$. Even though we are mostly interested in the compressed case $L<L_{0}$, we include the stretched case as well $\left(L>L_{0}\right)$. The bold line represents the aforementioned $L$-range, $0.5 L_{0}<L<1.2 L_{0}$. As shown in this figure, segregation is favored by free energy. This is consistent with our earlier finding that chains in the linear regime remain segregated. As $L$ decreases, the difference between the mixed and segregated cases becomes smaller. Our results in Figure 3 are, however, less accurate for $L<0.5 L_{0}$. Nevertheless, they suggest that the chains become more miscible as $2 L \rightarrow D$.

C. Bacterial Chromosomes and Confined Polymers. How much do chromosomes resemble polymer chains discussed in this work? When measured by fluorescence correlation spectroscopy, ${ }^{31}$ an isolated E. coli chromosome appears to consist of "structural units", each containing supercoiled plectonemes. ${ }^{31,32}$ Because of their topological structure, they should repel one another and can be considered as effective monomers; ${ }^{6}$ each monomer contains DNA segments and bound proteins. This mapping is based on the view that the action of DNA-binding proteins is local, ${ }^{3}$ and its effect can thus be subsumed into monomers. ${ }^{6}$ (See ref 33 for various DNA-binding proteins such as MukB.) The global properties of such chain molecules such as their interchain organization will not depend sensitively on this simplification, owing to chain connectivity. ${ }^{21}$ (See ref 34 for a similar coarsegrained, polymer model of eukaryotic chromosomes and ref 35 for coarse-graining in a more general context.)

If we use typical $E$. coli parameters such as the nucleoid diameter $D \approx 5$ and length $L \approx 28,{ }^{36}$ and the number of units $N \approx 200$ (see also refs $3,6,31$, and 32 ), we find $\xi \approx 3$; here all the lengths are given in units of the size of the structural unit $(\approx 70 \mathrm{~nm})$. The E. coli chromosome is indeed in de Gennes' linear ordering regime; i.e., it is weakly compressed or $\zeta_{\|} \approx \xi \approx$ $D$ with its degree of ordering similar to that illustrated in Figure 3 (see also Figure 5 in ref 6, which shows how ring topology enhances linear ordering). E. coli chromosomes in a dividing cell can segregate spontaneously. This is driven by physical effects chain molecules experience under anisotropic confinement. It is thus expected to play a nontrivial role in the global organization of chromosomes, largely independent of their local properties.

\section{DISCUSSION}

In conclusion, we have presented a physical picture of how interchain organization is related to intrachain ordering under cylindrical confinement, which has been illusive in the literature. Our simulations presented here suggest that while linear ordering ensures complete or almost complete segregation, it is not required for segregation. Even when each chain consists of randomly packed blobs along its entire length, the chain is still restricted in the transverse direction (see Figure 1). This anisotropy is responsible for chain segregation up to $70-80 \%$, outside de Gennes' linear-ordering regime.

Earlier studies indicate that the E. coli chromosome is linearly ordered. ${ }^{23}$ In light of our results reported here, it is clear that $E$. coli chromosomes in a dividing cell are in the entropic segregation regime. Note that this conclusion is largely independent of such molecular details as ring topology and local packing of the chromosome. While the former effect can be accurately mimicked in the linear case by adjusting cylinder diameters, ${ }^{6}$ the latter can be subsumed into monomers in our polymer model, since we are interested in much large-scale properties. $^{21,35}$

Technical advances now allow one to isolate, confine, and manipulate single chromosomes in narrow channels. ${ }^{37,38}$ The interrelationship between intrachain ordering and interchain organization described in this work will be useful for further exploiting the entropic mechanism ${ }^{3-8}$ and can serve as a model for comparison with future experiments.

\section{AUTHOR INFORMATION}

\section{Corresponding Author}

*E-mail: yjung@kisti.re.kr (Y.J.); byha@uwaterloo.ca (B.-Y.H.).

Notes

The authors declare no competing financial interest.

\section{ACKNOWLEDGMENTS}

B.-Y.H. acknowledges the financial support of NSERC (Canada). We thank C. Jeon for helpful discussions. This work was in part supported by the NRF of Korea funded by the MEST (No. 2011-0028908) (J.K.).

\section{REFERENCES}

(1) Kleckner, N.; Zickler, D.; Jones, G. H.; Dekker, J.; Padmore, R.; Henle, J.; Hutchinson, J. A mechanical basis for chromosome function. Proc. Natl. Acad. Sci. U. S. A. 2004, 101, 12592-12597.

(2) Bloom, K.; Joglekar, A. Towards building a chromosome segregation machine. Nature 2010, 463, 446-456.

(3) Jun, S.; Wright, A. Entropy as the driver of chromosome segregation. Nature 2010, 6, 600-607.

(4) Jun, S.; Mulder, B. Entropy-driven spatial organization of highly confined polymers: Lessons for the bacterial chromosome. Proc. Natl. Acad. Sci. U. S. A. 2006, 103, 12388-12393.

(5) Jung, Y.; Ha, B.-Y. Overlapping two self-avoiding polymers in a closed cylindrical pore: Implications for chromosome segregation in a bacterial cell. Phys. Rev. E 2010, 82, 051926-1-051926-5.

(6) Jung, Y.; Jeon, C.; Kim, J.; Jeong, H.; Jun, S.; Ha, B.-Y. Ring polymers as model bacterial chromosomes: confinement, chain topology, single chain statistics, and how they interact. Soft Matter 2012, 8, 2095-2102.

(7) Cook, P. R.; Marenduzzo, D. Entropic organization of interphase chromosomes. J. Cell Biol. 2009, 186, 825-834. 
(8) Dorier, J.; Stasiak, A. Topological origins of chromosomal territories. Nucleic Acids Res. 2009, 37, 6316-6322.

(9) Similar effects can be induced by chain topology such as ring or branchlike structure (see for instance refs $6-8$ ). In particular, the recent theoretical and numerical studies suggest that under cylindrical confinement the miscibility of ring polymers can map onto that of an appropriately chosen linear chain case.

(10) Daoud, M.; de Gennes, P. G. Statistics of Macromolecular Solutions Trapped in Small Pores. J. Phys. (Paris) 1977, 38, 85-93.

(11) Teraoka, I.; Wang, Y. Computer simulation studies on overlapping polymer chains confined in narrow channels. Polymer 2004, 45, 3835-3843.

(12) Ptacin, J. L.; Lee, S. F.; Garner, E. C.; Toro, E.; Eckart, M.; Comolli, L. R.; Moerner, W. E.; Shapiro., L. A spindle-like apparatus guides bacterial chromosome segregation. Nat. Cell Biol. 2010, 12, 791-798.

(13) Toro, E.; Shapiro, L. Bacterial Chromosome Organization and Segregation. Cold Spring Harb. Perspect. Biol. 2010, 2, a000349-1a000349-15.

(14) Su, T.; Das, S. K.; Xiao, M.; Purohit, P. K. Transition between Two Regimes Describing Internal Fluctuation of DNA in a Nanochannel. PLoS One 2011, 6, e16890-1-e16890-9.

(15) Buenemann, M.; Lenz, P. Geometrical Model for DNA Organization in Bacteria. PLoS One 2010, 5, e138061-e13806-13.

(16) Wang, Y.; Tree, D. R.; Dorfman, K. D. Simulation of DNA Extension in Nanochannels. Macromolecules 2011, 44, 6594-6604.

(17) Milchev, A. Single-polymer dynamics under constraints: scaling theory and computer experiment. J. Phys.: Condens. Matter 2011, 23, 1031011-1031011-24.

(18) Arnold, A.; Bozorgui, B.; Frenkel, D.; Ha, B.-Y.; Jun, S. Unexpected relaxation dynamics of a self-avoiding polymer in cylindrical confinement. J. Chem. Phys. 2007, 127, 164903-1164903-9.

(19) Jung, Y.; Jun, S.; Ha, B.-Y. Self-avoiding polymer trapped inside a cylindrical pore: Flory free energy and unexpected dynamics. Phys. Rev. E 2009, 79, 061912-1-061912-8.

(20) Jun, S.; Thirumalai, D.; Ha, B.-Y. Compression and Stretching of a Self-Avoiding Chain in Cylindrical Nanopores. Phys. Rev. Lett. 2008, 101, 138101-1-138101-4.

(21) de Gennes, P.-G. Scaling Concepts in Polymer Physics; Cornell University Press: Ithaca, NY, 1979.

(22) Nielsen, H. J.; Li, Y.; Youngren, B.; Hansen, F. G.; Austin, S. Progressive segregation of the Escherichia coli chromosome. Mol. Microbiol. 2006, 61, 383-393.

(23) Wiggins, P. A.; Cheveralls, K. C.; Martin, J. S.; Lintner, R.; Kondev, J. Strong intranucleoid interactions organize the Escherichia coli chromosome into a nucleoid filament. Proc. Natl. Acad. Sci. U. S. A. 2010, 107, 4991-4995.

(24) Weeks, J. D.; Chandler, D.; Andersen, H. C. Role of Repulsive Forces in Determining the Equilibrium Structure of Simple Liquids. J. Chem. Phys. 1971, 54, 5237-5247.

(25) Kremer, K.; Grest, G. S. Dynamics of entangled linear polymer melts: A molecular-dynamics simulation. J. Chem. Phys. 1990, 92, 5057-5086.

(26) Our date fitting analysis indicates that the slope of the RW regime is slightly smaller than $1 / 2$. Earlier it was shown that polymer conformations in a melt deviate from what we expect from the Flory's ideality hypothesis (see: Wittmer, J. P.; et al. Europhys. Lett. 2007, 77, 56003-1-56003-5 ). As a result, chain segments repel each other, at short length scales. This is not seen in our results, possibly because of confinement effects and the essential difference between the meltchain and confined-chain cases. In the latter case, the chain maps onto an equivalent semidilute solution ${ }^{27}$.

(27) Jun, S.; Arnold, A.; Ha, B.-Y. Confined Space and Effective Interactions of Multiple Self-Avoiding Chains. Phys. Rev. Lett. 2007, 98, 128303-1-128303-4.

(28) By themselves, two chains in a good or athermal solvent repel each other and are not miscible if in free space. Under confinement, this repulsion competes against compression, which tends to mix the chains. The resulting effective interaction dictates the effective miscibility measured in terms of $\lambda / L$. For our convenience, we use "effective miscibility" and "miscibility" interchangeably.

(29) Jacobsen, J. L. Demixing of compact polymer chains in three dimensions. Phys. Rev. E 2010, 82, 051802-1-051802-6.

(30) Vettorel, T.; Grosberg, A. Y.; Kremer, K. Statistics of polymer rings in the melt: a numerical simulation study. Phys. Biol. 2009, 6, 025013-1-025013-10.

(31) Romantsov, T.; Fishov, I.; Krichevsky, O. Internal Structure and Dynamics of Isolated Escherichia coli Nucleoids Assessed by Fluorescence Correlation Spectroscopy. Biophys. J. 2007, 92, 28752884.

(32) Woldringh, C. L.; Odijk, T. Structure of DNA within the Bacterial Cell: Physics and Physiology. In Organization of the Prokaryotic Genome; Charlebois, R. L., Ed.; ASM Press: Washington, DC, 1999

(33) Hirano, T. At the heart of the chromosome: SMC proteins in action. Nat. Rev. Mol. Cell Biol. 2006, 7, 311-322.

(34) Marko, J. F.; Siggia, E. D. Polymer Models of Meiotic and Mitotic Chromosomes. Mol. Biol. Cell 1997, 8, 2217-2231.

(35) Hyeon, C.; Thirumalai, D. Capturing the essence of folding and functions of biomolecules using coarse-grained models. Nat. Commun. 2011, 2, 487-1-487-11, DOI: $10.1038 /$ ncomms1481.

(36) The values refer to the nucleoid, i.e., the space inside which the chromosome is confined dominantly by molecular crowding. ${ }^{3,6,32}$

(37) Rasmussen, K. H.; Marie, R.; Lange, J. M.; Svendsen, W. E.; Kristensena, A.; Mir, K. U. A device for extraction, manipulation and stretching of DNA from single human chromosomes. Lab Chip 2011, 11, 1431-1433.

(38) Prinz, C.; Tegenfeldt, J.; Austin, R.; Cox, E.; Sturm, J. Bacterial chromosome extraction and isolation. Lab Chip 2002, 2, 207-212. 